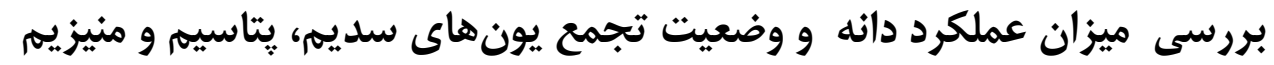

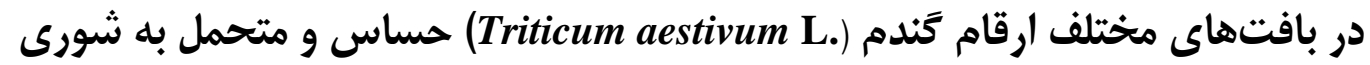

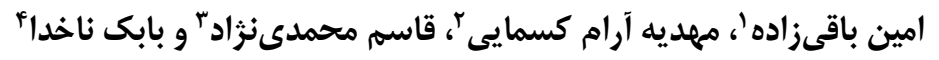

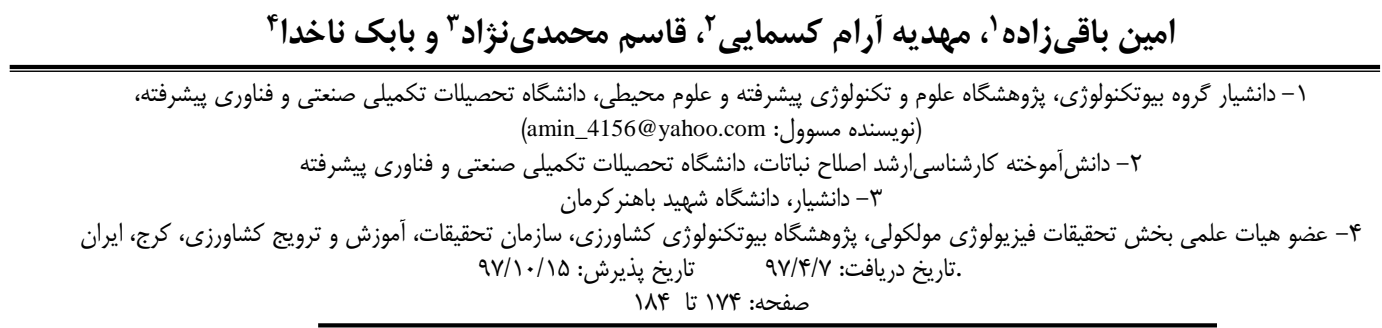

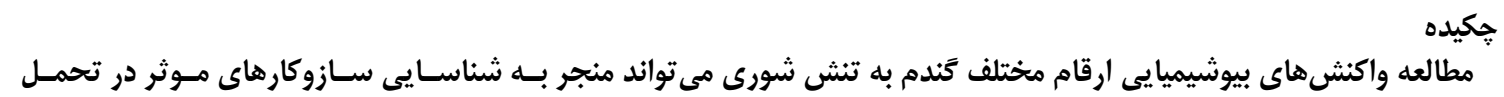

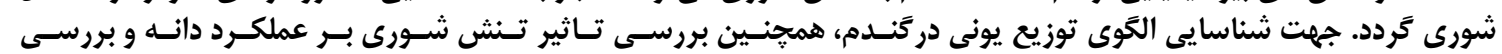

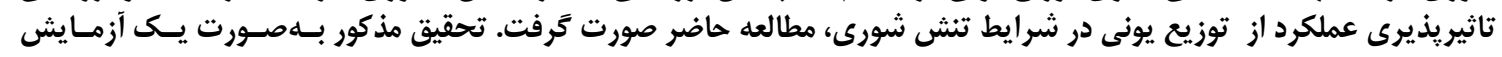

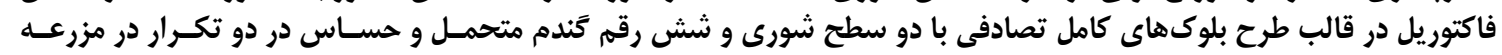

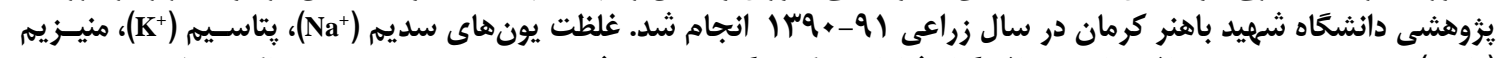

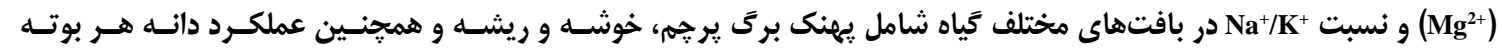

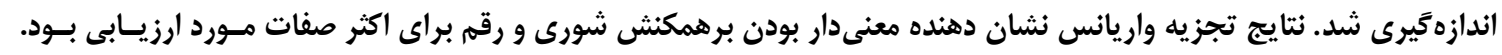

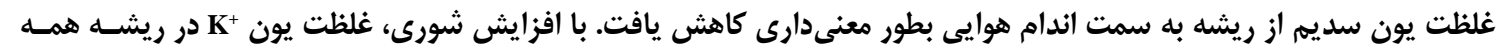

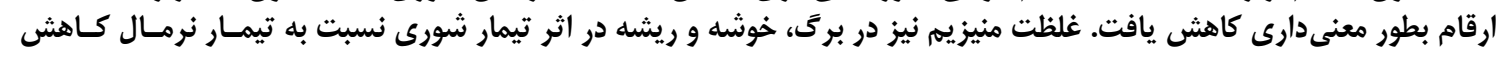

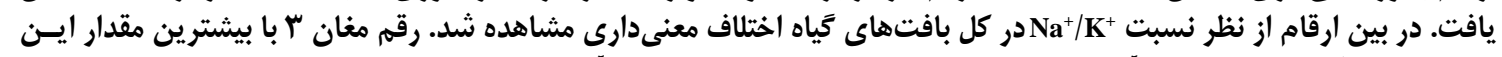

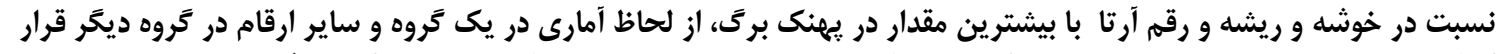

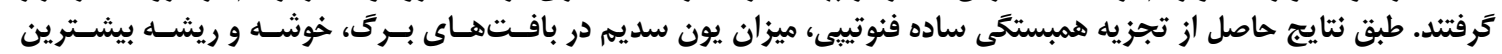

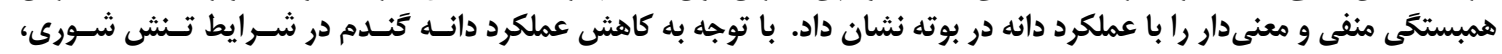

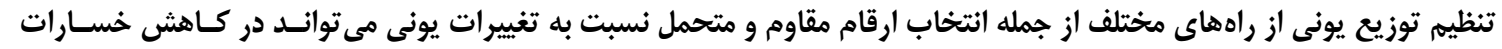
ناشى از تنش شورى موثر باشد.

وازههاى كليدى: تنثى شورى، غلظت يونى، عملكرد دانه، تحمل شورى

مىتواند باعث كاهش فعاليت فتوسنتزى شود كه در نهايت

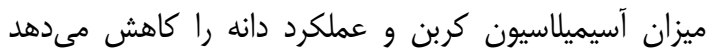

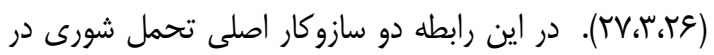
كياهان شامل انتقال مقادير يايينى از يون

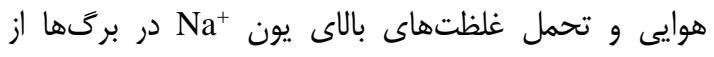

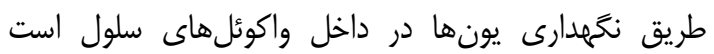

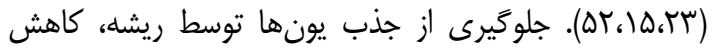

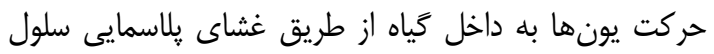

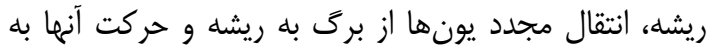

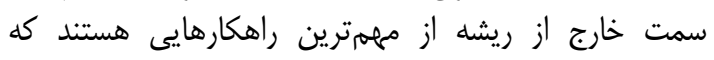

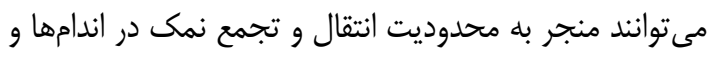

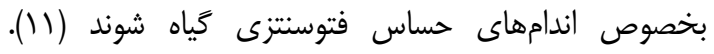

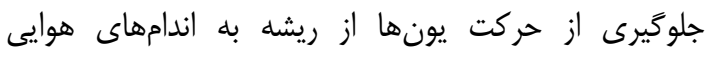

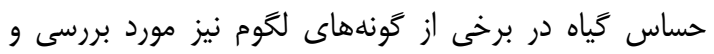

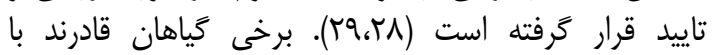

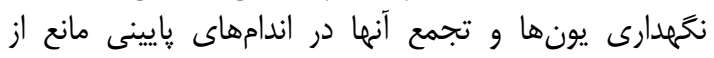

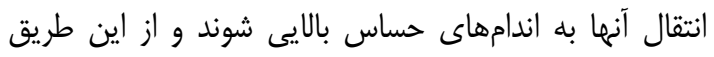

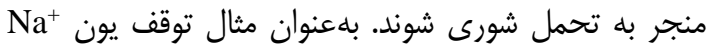

مقدمه گندم از جمله محصولات زراعى مهم است كه در بيشتر

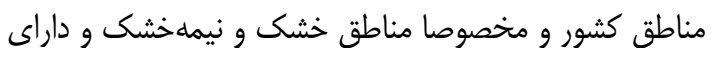

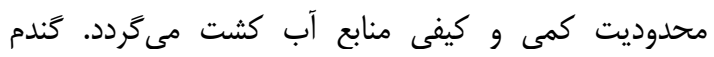

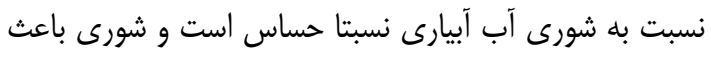

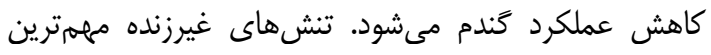

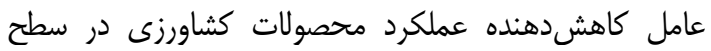

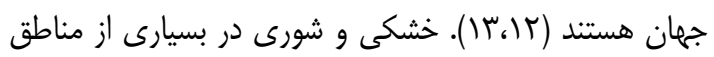

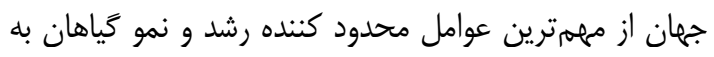
شمار مىروند، يك سازوكار مناسب در اين ماين زمينه، استفاده از

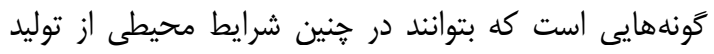

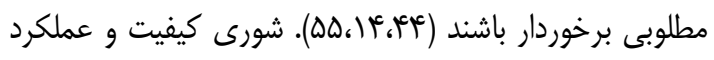

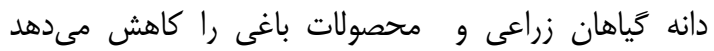

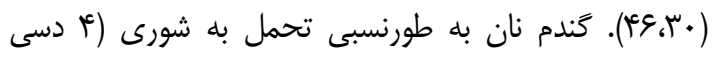

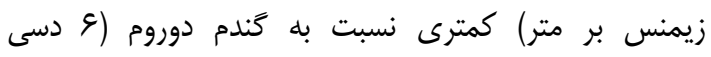

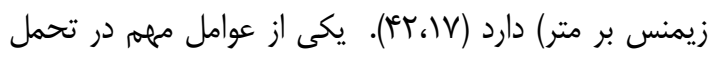

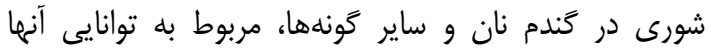

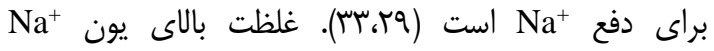




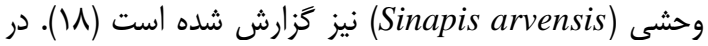

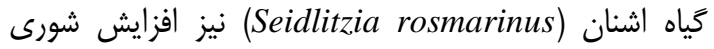

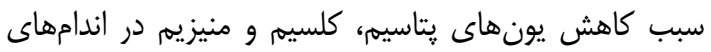

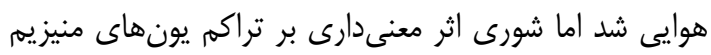

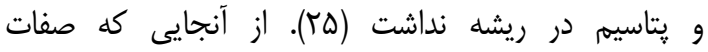

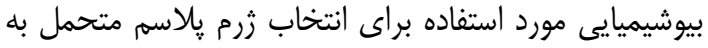

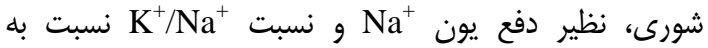

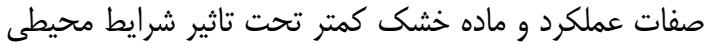

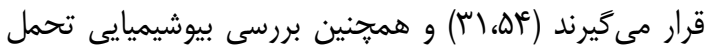

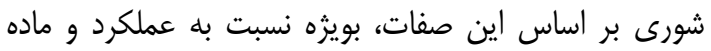

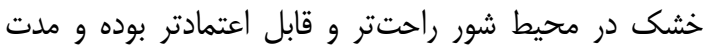

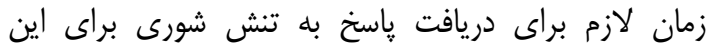

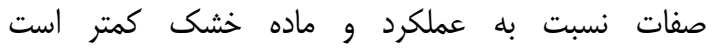

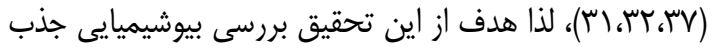

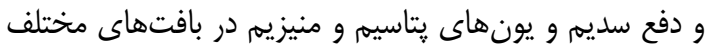

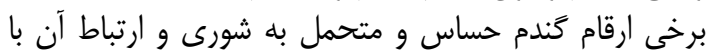

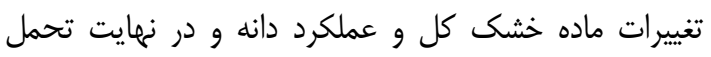

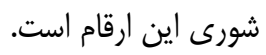

مواد و روشها محل و نحوه اجر اي آزمايش

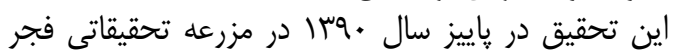

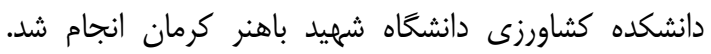

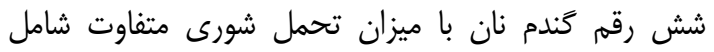

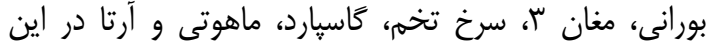

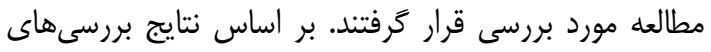

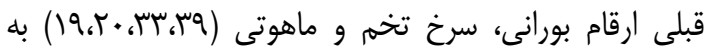

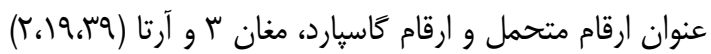

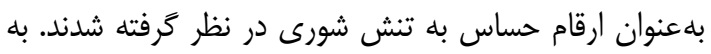

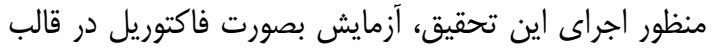

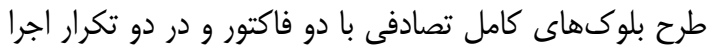

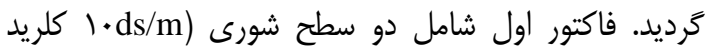

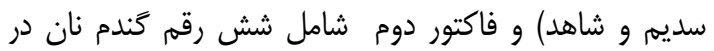

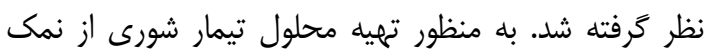

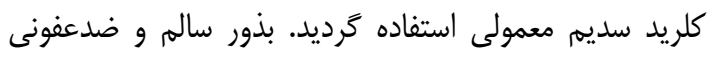

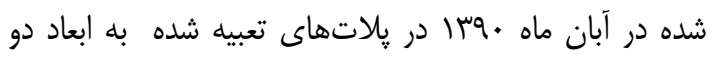

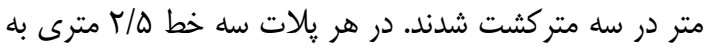

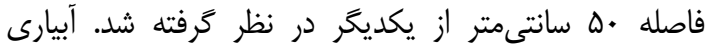

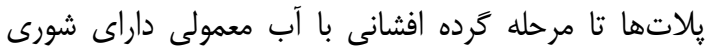

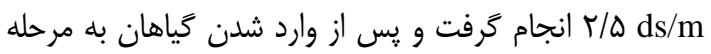

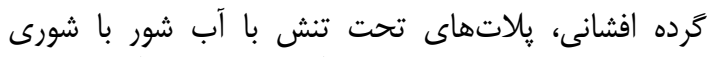

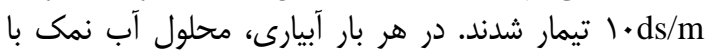

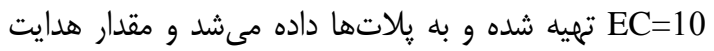

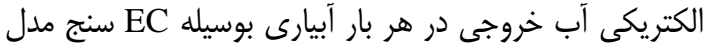

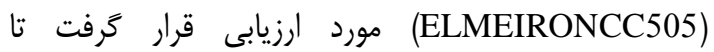
يلاتهاى تحت تنش در EC مورد نظر قرار بخيرند.
در ريشه، بركَها و غلاف برك تراس ها از جمله سازوكارهاى

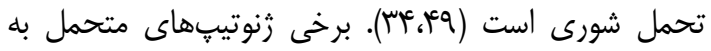

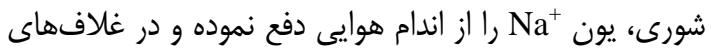

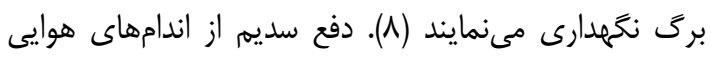

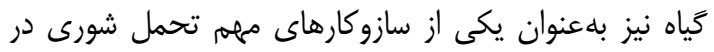

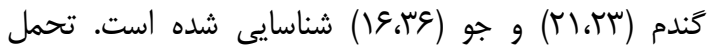

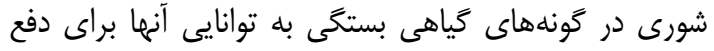
يون

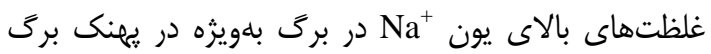

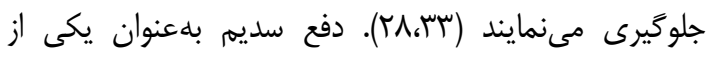

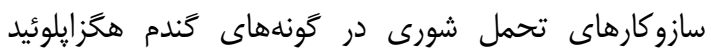

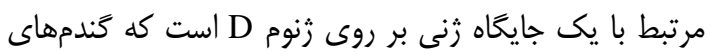

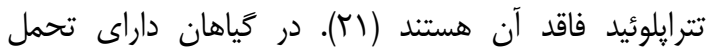
شورى، يونهاى

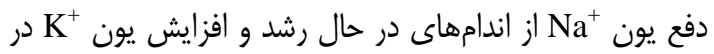

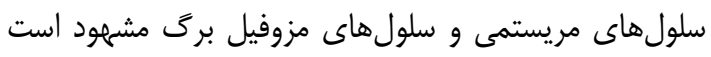

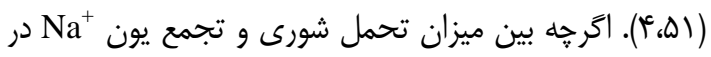

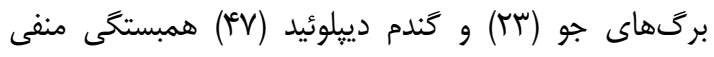

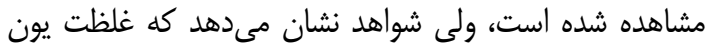

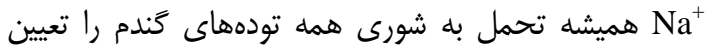

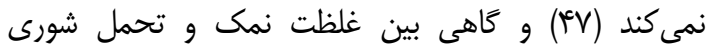

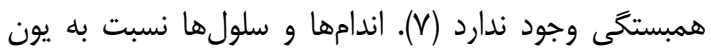

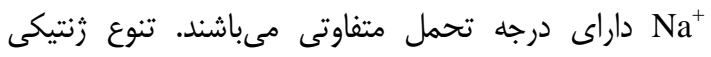

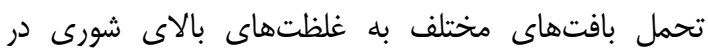

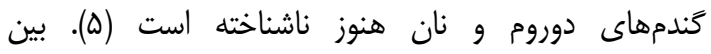

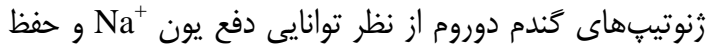

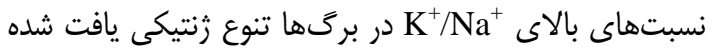

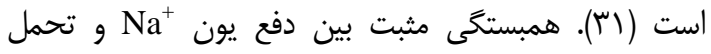

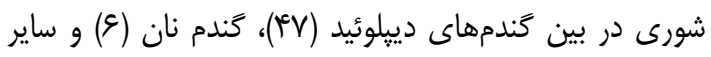

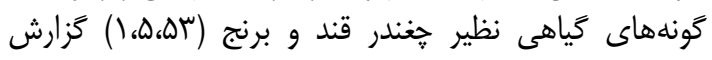

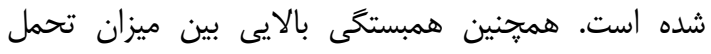

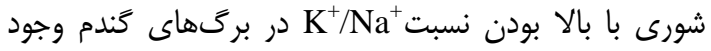

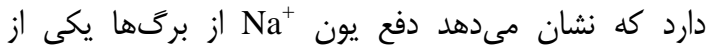

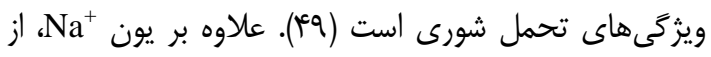

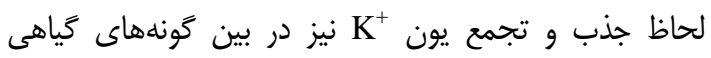

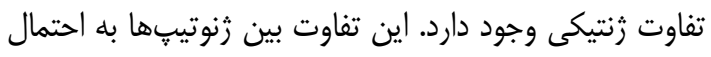

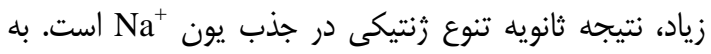

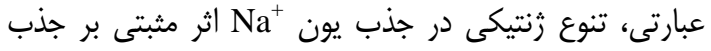

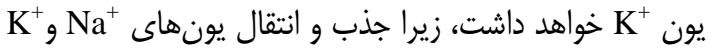

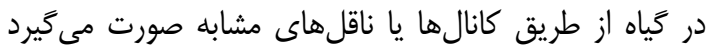

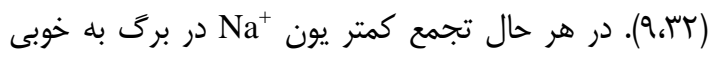

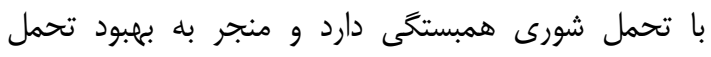

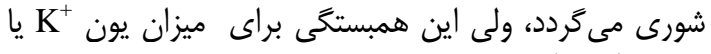

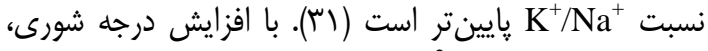
معمولا ميزان اين وضعيت در آزمايش قادريان و همكاران در روى خرى خردل 
رشد شديدتر از اثرات تنش يونى در داخل گياه است

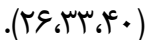

غلظت يون

سديم كاتيون اصلى است كه با افزايش شورى در بافتها و

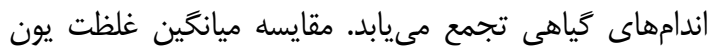

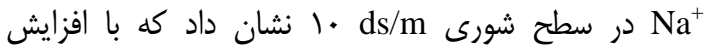

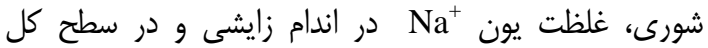

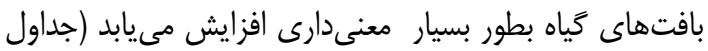

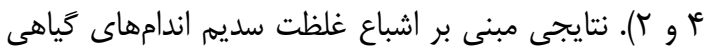

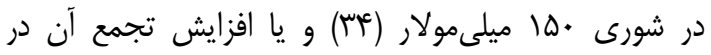

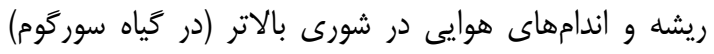

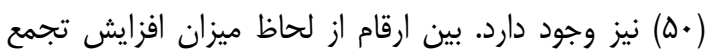

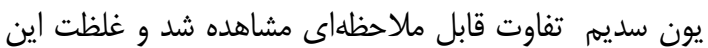

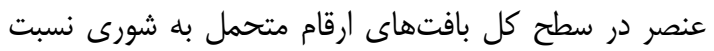

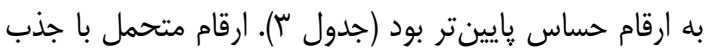

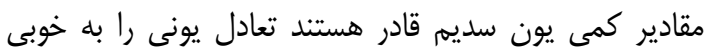

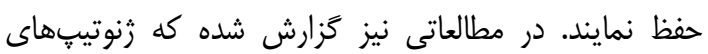

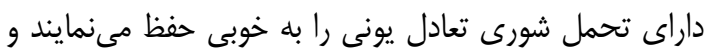

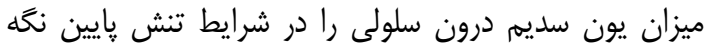

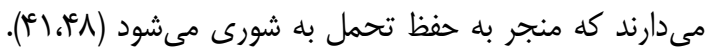

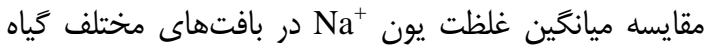

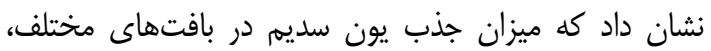

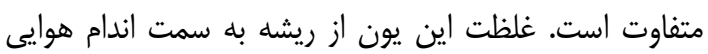

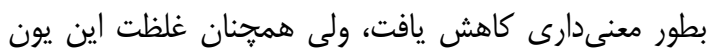

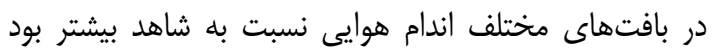

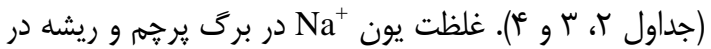
بالاترين مقدار بود و مقدار اين يون در اندام زايشى به به نسبت

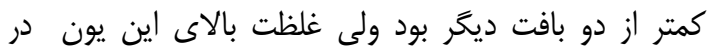

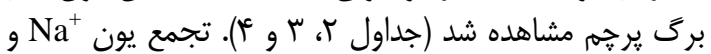

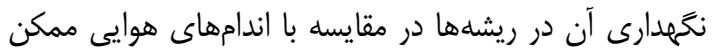

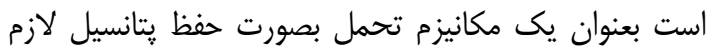

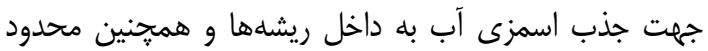

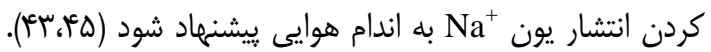

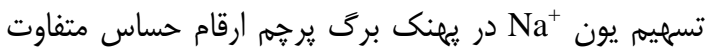

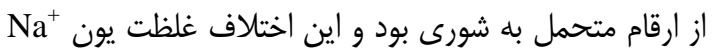

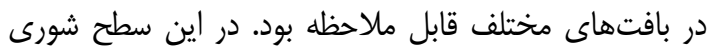

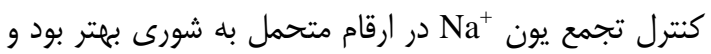

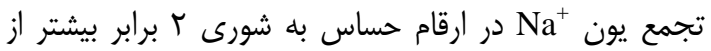

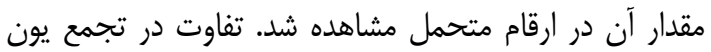

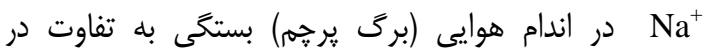

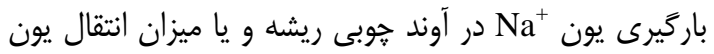
ل $\mathrm{Na}^{+}$

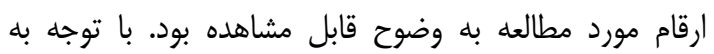

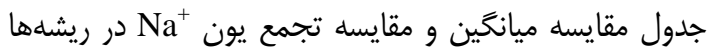

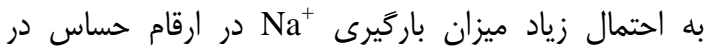
مقايسه با ارقام متحمل بيشتر اشد (جداد ميز باركي س). اين تفاوت حسات
اندازهَيرى عملكرد و غلظت يونها

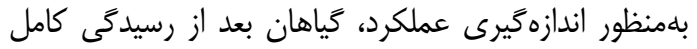

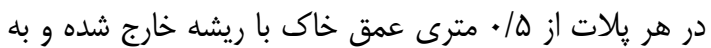

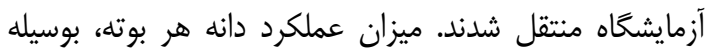

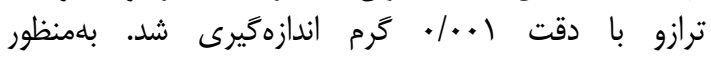
اندازهيرى غلظت يونها در بافتهاى مختلف خياه، يك ماه

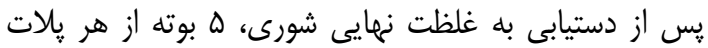

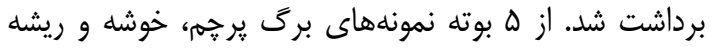

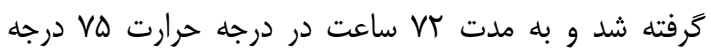

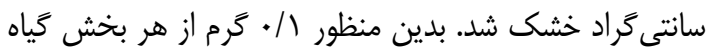

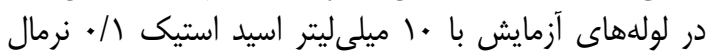

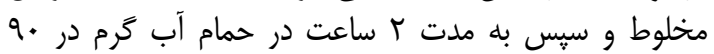

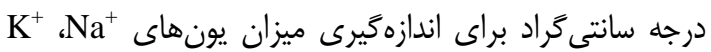
وستخاه $\mathrm{Mg}^{2+}$

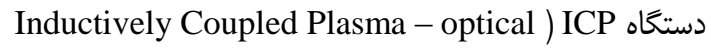
(emission spectrometer به يتاسيم در بافتهاى مختلف محاسبه شد. براى تجزيه

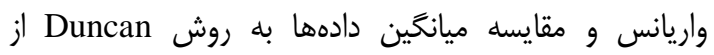
نرمافزار SAS نسخه

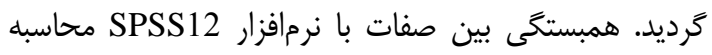

\section{نتايج و بحث}

اثر تنش شورى بر صفات مورد بر رسى در ارقام مختلف

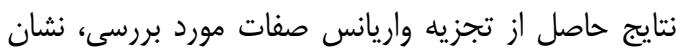
داد كه برهمكنش تنش شورى با ارقام براى كليه صفات مات مورد

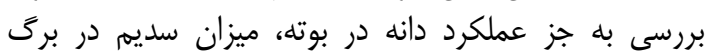

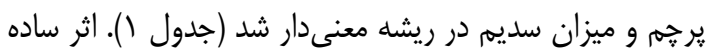

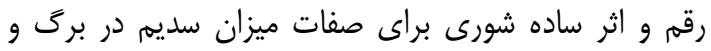

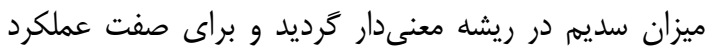

دانه در بوته تنها اثر ساده شورى معنى دار دار شد (جدول () ).

عملكرد دانه

مقايسه ميانگين عملكرد دانه در بوته تحت اثر دانه تيمار شورى

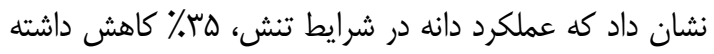

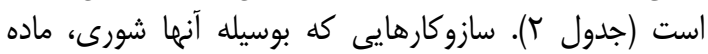

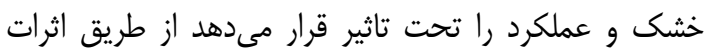

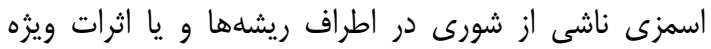

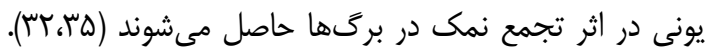

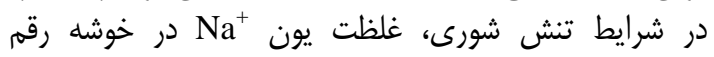

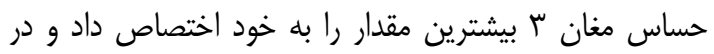

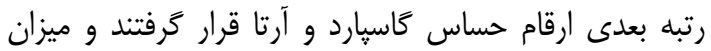

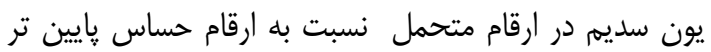

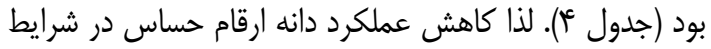

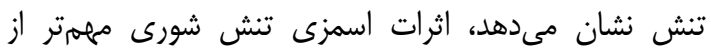

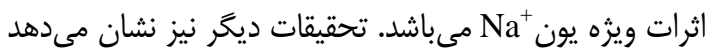

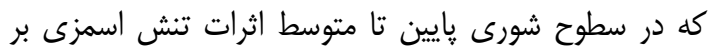


كياهان است تا انتقال يون مa به داخ ماخل سلولهاى

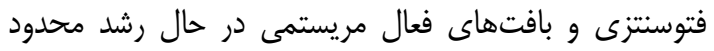

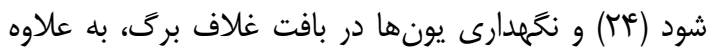

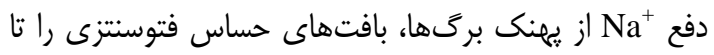

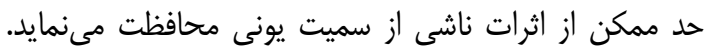

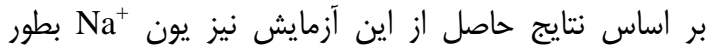

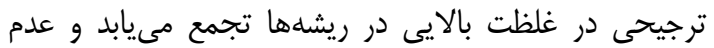

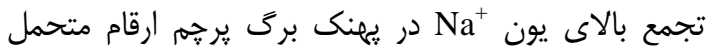

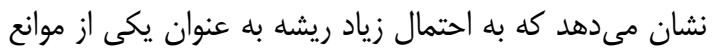

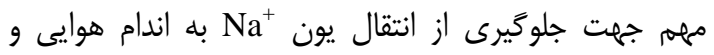

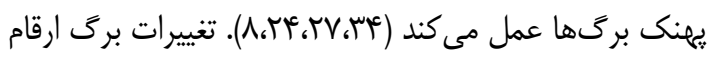

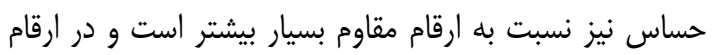

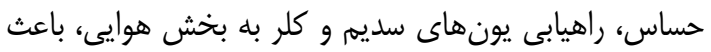

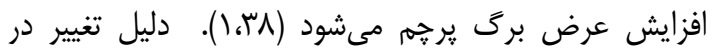

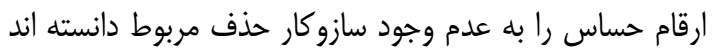

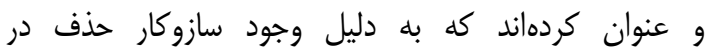

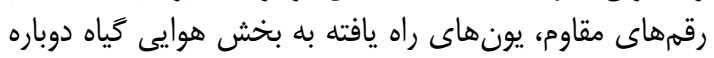

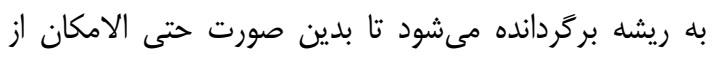

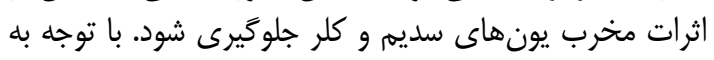

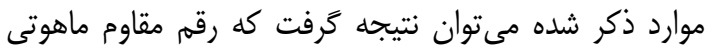

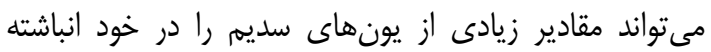

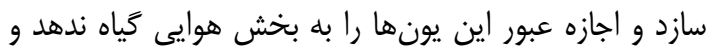
بدين ترتيب بخشهاى هوائ هوايى خود را از اثرات هوات سمى اين يونها مصون نغَه دارد.
زنتيكى در ميزان باركيرى يون

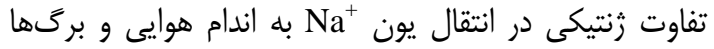

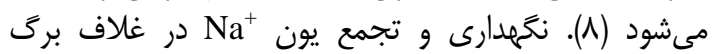

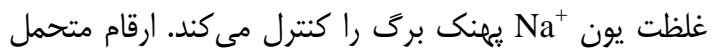

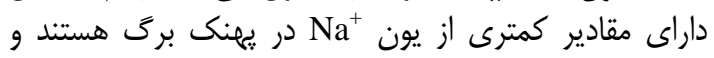

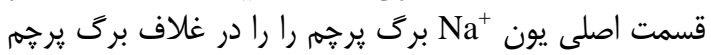

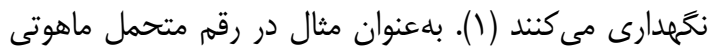
محتواى

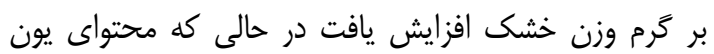

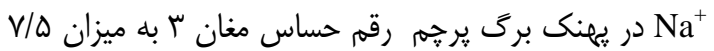

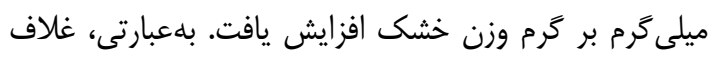

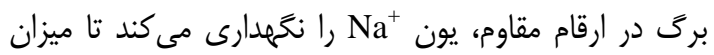

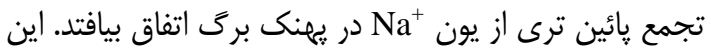

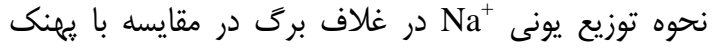

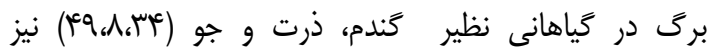

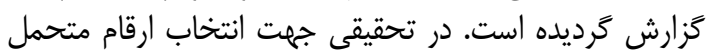

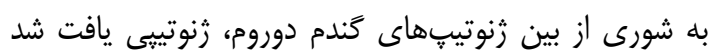

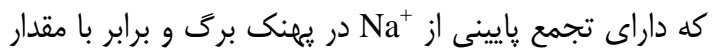

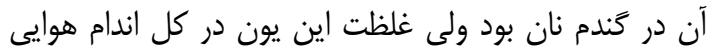

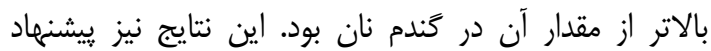

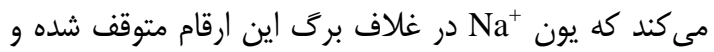

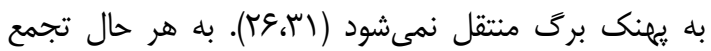

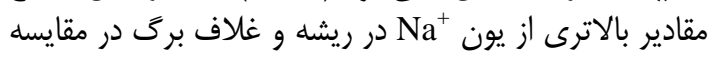

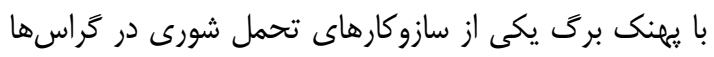
است و يكى از عوامل تعيين كننده تحمل تنش شازئ شورى درى در اين 
Table 1. Mean squares obtained from analysis of variance of studied traits

جدول 1- ميانگين مربعات حاصل از تجزيه واريانس صفات مورد ارزيابى

\begin{tabular}{|c|c|c|c|c|c|c|c|c|}
\hline \multicolumn{9}{|c|}{ ميانكين مربعات } \\
\hline ميزان منيزيم در برك يرجهم & ميزان يتاسيم در ريشه & ميزان يتاسيه در خوشه & ميزان يتاسيم در برك يرجهم & ميزان سديم در ريشه & ميزان سديم در خوشه & ميزان سديم در برك يرجم & df & منابع تغيير \\
\hline$\Delta / \backslash \Lambda$ & rGY/•ᄉ & $r Q N / \Delta \Lambda$ & TTVT/Tq &.$/ \pi$ & $1 / \pi r$ & $\cdot / \pi \& \wedge$ & 1 & تكلا \\
\hline rצY /9** & Varo/rus & 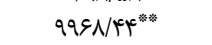 & $M M Y I . \varphi^{\circ}$ & rq/.r䋛 & 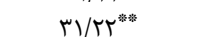 & $|F| / \uparrow^{*} \Lambda^{* * 3}$ & 1 & ش ش شرو \\
\hline $1 r q / \mathcal{N}^{* * * a s}$ & IfDQT/Fe" & $19 \cdot r \mu / \mu^{2}$ & $\mid \& \mu \& \Delta / V^{* * *}$ & $\Delta / \wedge \mathcal{V}^{*}$ & $1 / 94^{*}$ & $9 / 4 V^{*}$ & $\Delta$ & سوقم \\
\hline$G T / \mu Y^{*}$ & $9 \wedge 9 \cdot / \Delta r^{*}$ & 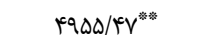 & IVร99/V| & $r / \Delta \omega$ & $r / q \mu^{*}$ & $r / 4 q$ & $\Delta$ & رقم ششورى \\
\hline 1.1 .4 & $\| r \mid M$ & IVT/IT & $1 \cdot \Delta \& / \Delta \mid$ &.$/ 9 \Delta$ &.$/ 4 \Delta$ & $1 / 4 \cdot r$ & 11 & خط \\
\hline $11 / 91$ & $\mathrm{IV} / \mathrm{WV}$ & s/ar & $\mid 81.9$ & $r V / \cdot 1$ & $1 V / 9$ & $r \Delta / 9 \varphi$ & - & $\mathrm{CV}$ \\
\hline
\end{tabular}

\begin{tabular}{|c|c|c|c|c|c|c|c|}
\hline \multicolumn{8}{|c|}{ Continue Table1. Mean squares obtained from analysis of variance of stud } \\
\hline عملكرد دانه هر بوته & نسبت سديم به يتاسيم در ريشه & نسبت سديم به يتاسيم در خوشه & 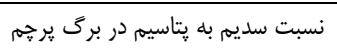 & ميزان منيزيم در ريشه & ميزان منيزيم در خوشه & df & منابع تغيير \\
\hline $1 \cdot \Delta / r^{*}$ &.$/ \cdots \varphi^{\alpha}$ & $\cdot / \cdots \cdots \mathrm{V}^{*}$ &.$/ \cdots \cdots 19$ & $r / r \Delta$ & $11 / v \wedge$ & 1 & تكرار \\
\hline 每 & $. \cdot . r^{* * *}$ &.$/ . r \Lambda^{* * *}$ &.$/ .199^{* * * * * 3}$ & 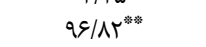 & $\Lambda \mathrm{M} / \mathrm{r}^{* *}$ & 1 & 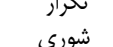 \\
\hline G/ &.$/ \ldots \mathrm{vq}^{\text {mat }}$ &.$/ \cdots \Delta e^{n * m}$ &.$/ \cdots \Delta q^{* * *}$ & $1.918^{* * *}$ & $1 . r / r^{* * *}$ & $\Delta$ & ( ) \\
\hline$r / l r$ &.$\ldots r \Delta^{*}$ & $\cdot / \ldots \varphi^{\text {w** }}$ &.$/ \ldots r^{*}$ & $\mathrm{FV} / \mathrm{Tr}$ & 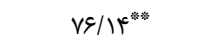 & $\Delta$ & إقم×شوري \\
\hline $1 / 9 \cdot 4$ & $. \ldots . .94$ &.$/ \ldots \cdot 1 \Delta$ & $\%$. Nor & $F / \Delta S$ & $\Delta / \Delta F$ & 11 & 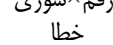 \\
\hline W/91 & ו & $10 / 49$ & TN/DI & $V / V I$ & Q/Fr & - & $\mathrm{CV}$ \\
\hline
\end{tabular}

Table 2. Comparison of mean simple effects of salinity for evaluated traits

جدول r- مقايسه ميانخين اثرات ساده شورى براى صفات مورد ارزيابى

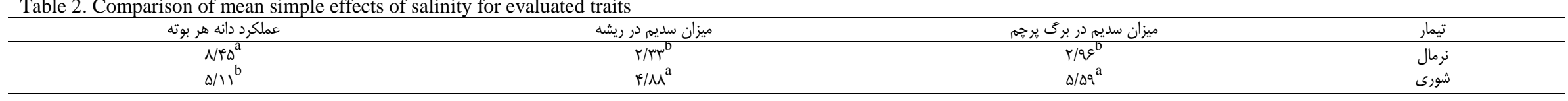

جدول ب- مقايسه ميانگين اثرات ساده رقم براى صفات مورد ارزيابى

Table 3. Comparison of mean simple effects of the cultivar for the evaluated traits

\begin{tabular}{|c|c|c|}
\hline ميزان سديم در ريشه & ميزان سديم در برك يرجمه & رقم \\
\hline$r / \cdot \Lambda^{\mathrm{bc}}$ & $r / T v^{b c}$ & سرخ تخم \\
\hline$\left.r / c^{c}\right)^{\mathrm{ab}}$ & $r / q q^{a b}$ & كاسيارد \\
\hline$\Gamma / / \mathrm{rc}^{\mathrm{bc}}$ & $\Delta / \cdot r^{\mathrm{ab}}$ & آرتا \\
\hline
\end{tabular}


Table 4. Comparison of mean for cultivar interaction in salinity for evaluated traits

جدول F- مقايسه ميانحين براى برهمكنش رقم در شورى در مورد صفات مورد ارزيابى

\begin{tabular}{|c|c|c|c|c|c|c|c|c|c|c|c|}
\hline نسبت سديم به يتاسيم & نسبت سديم به يتاسيم & نسبت سديم به پِتاسيم & ميزان منيزيم در & خيزان منيزيم در & ميزان منيزيم در برى & ميزان يتاسيم در & خيزان يتاسيم در & ميزان يَاسيم در & خيزان سديم در & رقم & تيمار \\
\hline $.1 \cdot 1.99^{\mathrm{cd}}$ & $.1 \cdot 19 M^{\mathrm{de}}$ & $.1 .11999^{d}$ & $r g / r^{\text {cde }}$ & $r \mu / r \cdot \operatorname{def}$ & $r \omega / . r^{b c d}$ & $r / r^{b c}$ & $\mid V \Delta / \mathrm{f}^{\mathrm{c}}$ & $\mid r r / \Lambda^{c d}$ & $r / q \uparrow q^{\mathrm{de}}$ & بورانى & \\
\hline$\cdot / \cdot r \cdot \Delta f^{c d}$ & . $/ .1 \% \pi \Delta^{\operatorname{def}}$ & $\cdot / \cdot r \cdot v^{c d}$ & $r V / \Delta \cdot^{a}$ & $r T / Y \cdot{ }^{a b}$ & $r V / \uparrow \Lambda^{a}$ & $|F q /|^{c d}$ & $\Lambda \mu r / r^{c}$ & $189^{\mathrm{cd}}$ & $r / 4 r \varepsilon^{e}$ & مغان r & \\
\hline . . . & $\cdot / \cdot \Delta s+q^{f}$ & $\cdot / \cdot v \Delta \cdot \Delta^{d}$ & $r \cdot / 98^{\mathrm{bc}}$ & $10 / \Delta 9^{g}$ & $r \cdot / \Delta \Lambda^{\mathrm{ab}}$ & $r \wedge q / \Lambda^{a}$ & $r \Lambda r / r^{a}$ & 年 & $r /|\varepsilon|^{e}$ & سرخ تخم & \\
\hline.$/ \cdot 191 \mathrm{r}^{\mathrm{cd}}$ &.$/ .11 \mathrm{M}^{\mathrm{ef}}$ &.$\left.|\cdot r| \varepsilon\right|^{c d}$ & $r F / v)^{a b}$ & $r / \Delta \omega^{a}$ & $r \omega / q \varphi^{a}$ & $|Q| / \Lambda^{\mathrm{cd}}$ & $\mid \mathrm{W} / \mathrm{r}^{\mathrm{c}}$ & $\mid c F / \mu^{c d}$ & $r / 1.9^{e}$ & كاسيارد & ن لرمال \\
\hline$\cdot / \cdot 1 \cdot \mathrm{M}^{\mathrm{cd}}$ & $.1 \cdot r r \Delta r^{d}$ & $\cdot / \cdot 1 \& 99^{d}$ & $r / / q^{\mathrm{efg}}$ & $r N / \cdot V^{b c d}$ & $r r / r r^{c d}$ & $199^{b c d}$ & $\mid r v / \Delta^{d}$ & $r \checkmark \cdot / \mu^{\mathrm{a}}$ & $\mu / / \mu q^{\text {de }}$ & ماهوتى & \\
\hline.$/ . q q \mathrm{Fr} \mathrm{cd}^{\mathrm{cd}}$ & 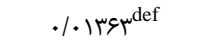 & & $r V / M \Lambda^{c d}$ & $r \Delta / \neg \lambda^{\text {cde }}$ & $r V / V e^{b c}$ & $\mid V V^{w} / \kappa^{b c d}$ & $r \cdot I / \Lambda^{c}$ & $\mid F \varphi / T^{\mathrm{cd}}$ & $r / V Q)^{\mathrm{de}}$ & آرتا & \\
\hline.$/ . \uparrow \& \Delta \gamma^{b c}$ &.$/ 10 r^{\text {de }}$ & $.1 \cdot 1 k g e^{d}$ & $19 / 9 \mathrm{rg}^{\mathrm{fg}}$ & $19 / \mu r^{f g}$ & $W / \mid \Psi^{\mathrm{de}}$ & $\mid F F / \Delta^{c d}$ & $r \cdot \Delta / \Delta^{c}$ & $r \Delta q / v^{b}$ & r/Iqude & بورانى & \\
\hline.$/ V e^{e}{ }^{a}$ & $.1 .911 r^{\mathrm{a}}$ &.$\cdot$ FVY $^{\mathrm{b}}$ & $r q / \xi^{c}$ & $r \cdot / v g^{\mathrm{efg}}$ & $T T^{c} / \varepsilon \Lambda^{\mathrm{bcd}}$ & $1 \cdot V / q^{d}$ & $1 . \Delta / r^{\mathrm{e}}$ & $119 / 9^{b c}$ & $V / \backslash M^{a}$ & مغان ب & \\
\hline $.1 .194 \mathrm{rd}^{\mathrm{cd}}$ & $.1 \cdot 19 \cdot 9^{\mathrm{de}}$ & $\cdot|\cdot r| \cdot q^{c d}$ & $r r / \Lambda)^{\text {def }}$ & r 1 def & $r I / r \cdot{ }^{c d}$ & $r \mid r / r^{b c}$ & $r \varepsilon a / \Lambda^{b}$ & $19 \cdot / 1^{b c}$ & $r / r \cdot \varphi^{c d}$ & سرخ تخم & \\
\hline . $1.4998^{b}$ & . & /. Mfef. bc & $r \Delta / v \varepsilon^{\text {cde }}$ & $r N / q \Lambda^{b c}$ & $r \Delta / \|^{\text {bcd }}$ & $\mid V Y^{c} / \mathrm{e}^{\mathrm{cd}}$ & $\mid r N / \kappa^{d}$ & $\mid \Lambda \mu^{\mu} \kappa^{\mathrm{bcd}}$ & $\Delta / \cdot \wedge \mu^{b c}$ & كاسيارد & 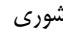 \\
\hline.$/ 1 r \Delta q^{c d}$ & $. / .1 M .^{\mathrm{de}}$ & ו וצr.|Cd & $|N /|^{e^{g}}$ & $1 \omega / T V^{g}$ & $I \Psi / V \Delta^{e}$ & 的/ & $194 / /^{c}$ & $\mid V \Delta / q^{c d}$ & 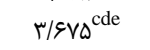 & ماهوتى & \\
\hline$\cdot / \cdot r \Delta \wedge \varepsilon^{b c d}$ &.$/ . \Delta 9 Y q^{b}$ & $.1 .944 \mathrm{qa}^{\mathrm{a}}$ & $r g /\left.g\right|^{a}$ & $\Gamma / / \omega^{\mathrm{ab}}$ & $r g / v)^{a}$ & $|V N /|^{\mathrm{bcd}}$ & $99 / \wedge r^{\mathrm{e}}$ & $1 \cdot F / T^{d}$ & $\Delta / 9 / f^{a b}$ & آرتا & \\
\hline
\end{tabular}

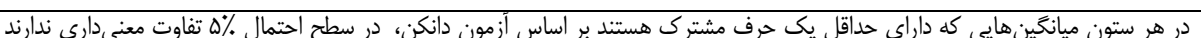




\section{نسبت $\mathrm{Na}^{+} / \mathrm{K}^{+}$}

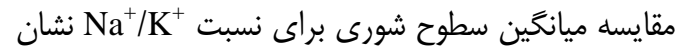

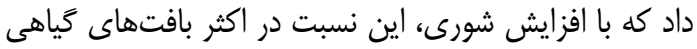

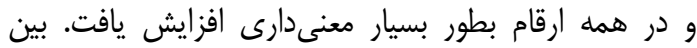

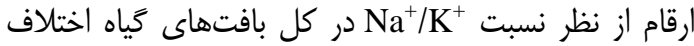

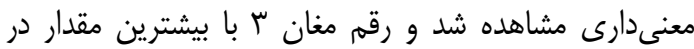

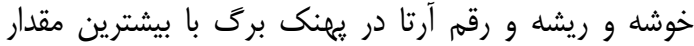

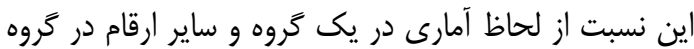

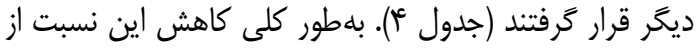

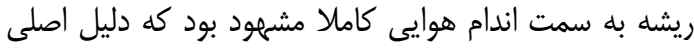

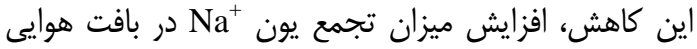

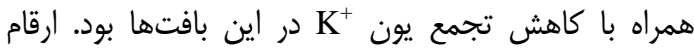

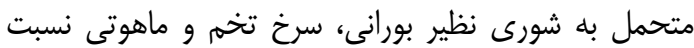

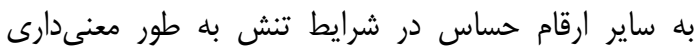

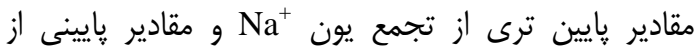

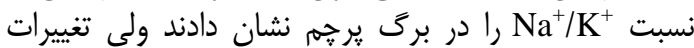
جندانى در غلظت يون

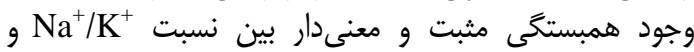

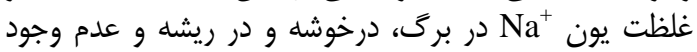

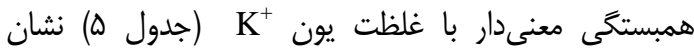
مىدهد كه ميزان تجمع يون معنى

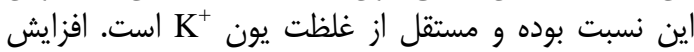

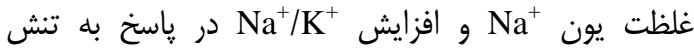

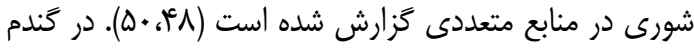

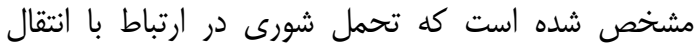

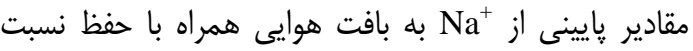

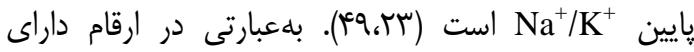
تحمل شورى ميزان تجمع كمتر يون يايين ترل

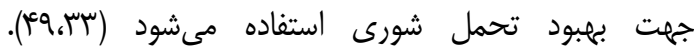

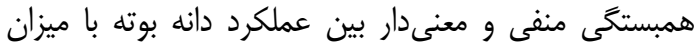

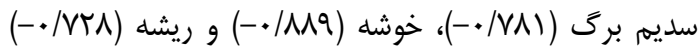

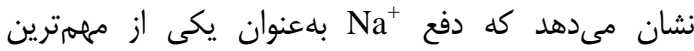

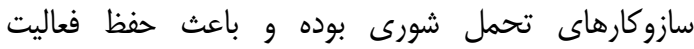

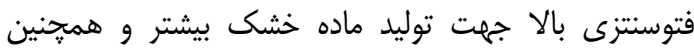

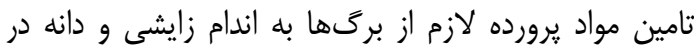

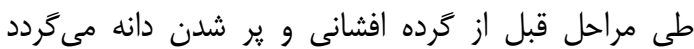

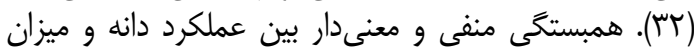

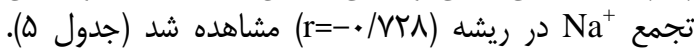

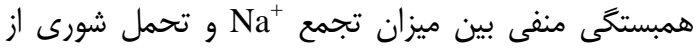

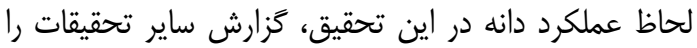

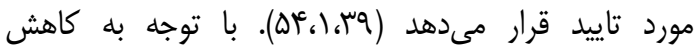

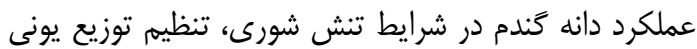

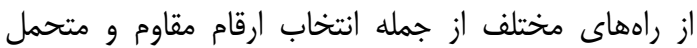

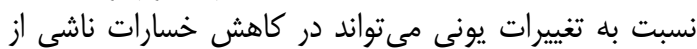
تنش شورى موثر باشد.
غلظت يون Ke

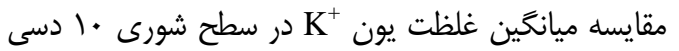

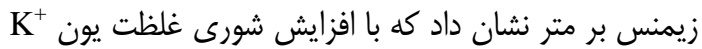

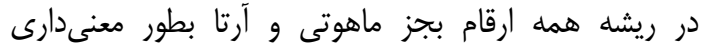

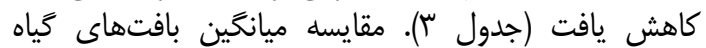

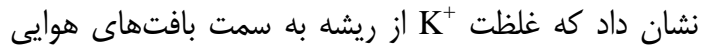

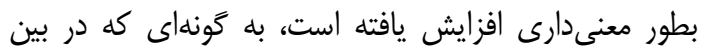

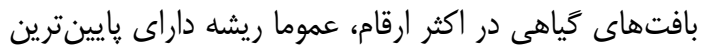

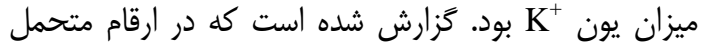

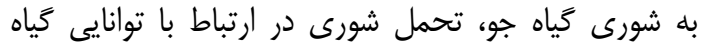

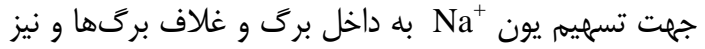

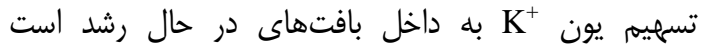

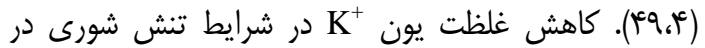

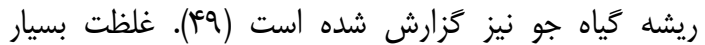
بالاى يون

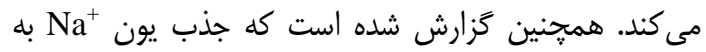

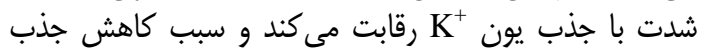

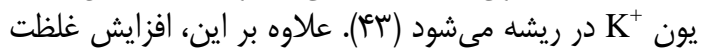

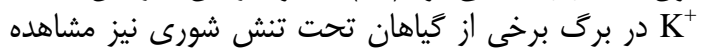

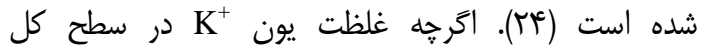

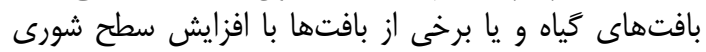

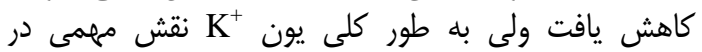

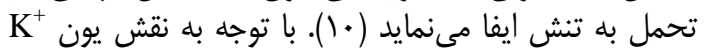

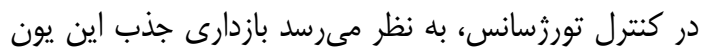

از رشد جلو عيرى مى كند (سأ). غلظت يون

غلظت يون منيزيم در تيمار شورى نسبت به به تيمار نرمال

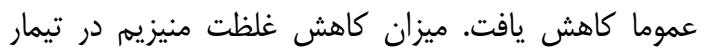

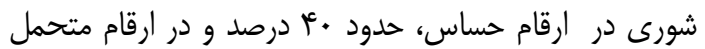

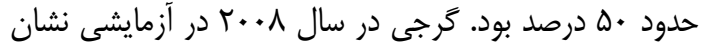

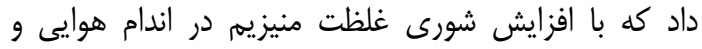

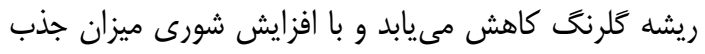

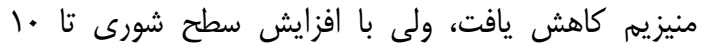

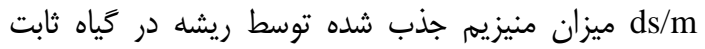

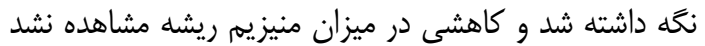

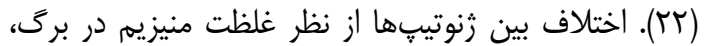

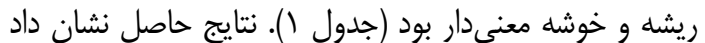

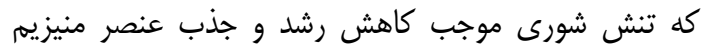

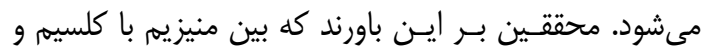

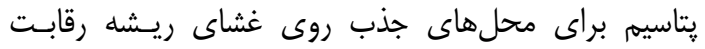

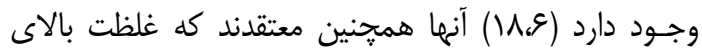
منيزيم در خاى ممكن است بـا ايجاد كمبود كلسيم در كَّياه،

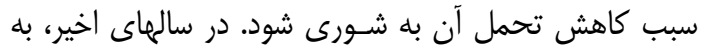

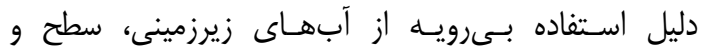

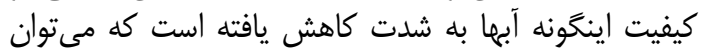
به برهمخوردن تعادل ميان عناصـر منيـزيم و كلسيهم به نفع إنه

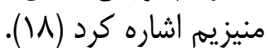


1. Asch, F., M. Dingkuhn, K. Dorffling and K. Miezan. 2000. Leaf K+/Na+ ratio predicts salinity in induced yield loss in irrigated rice. Euphytica, 113(2): 109-118.

2. Bandehhagh, A., H. Kazemi, M. Valizade and A. Javanshir. 2004. Salt tolerance of spring wheat (Triticum aestivum L.) cultivars during vegetative and reproductive growth. Iranian Journal of Agricultureal Sciences, 35(1): 61-71.

3. Bhatti, M.A., A. Zulfiqar, A. Razaq and A.R. Jamali. 2004. Screening of wheat lines for saliny tolerance. International Journal of Agriculture and Biology, 6: 627-628.

4. Boursier, P., J. Lynch, A. Lauchli and E. Epstein. 1987. Chloride partitioning in leaves of salt Stressd sorghum, maize, wheat and barley. Australian Journal of Plant Physiology, 14(4): 463 -473.

5. Cherki, G., A. Foursy and K. Fares. 2002. Effects of salt stress on growth, inorganic ions and prolin accumulation in relation to osmotic adjustment in five sugare beet cultivares. Environ and Exper. Botany, 47: 39-50.

6. Chhipa, B.R. and P. Lal.1995. Na/K ratios as the basis of salt tolerance in wheat. Australian Journal of Agricultural Research, 46(3): 533-539.

7. Cramer, G.R., G.L. Alberico and C. Schmidet. 1994. Salt tolerance is not associated with the sodium accumulation of two maize hybrids. Australian Journal of Plant Physiology, 21(5): 675-692.

8. Davenport, R., R.A. James, A. Zakrisson- Plogander, M. Tester and R. Munns. 2005.Control of sodium transport in durum wheat. Plant Physiology, 137(3): 807-818.

9. EL.Hendauy, S.E., H. Yuncai, G.M. Yakoutb, A.M. Awad, S.E. Hafiz and U. Schmidhater. 2005. Evaluating salt tolerance of wheat genotypes using multiple parameters. Europ. Journal Agron, 22: 243253.

10. Epstein, E. 1998. How calcium enhances plant salt tolerance. Science, 280(5371), 1906-1907.

11. Erdei, L. and E. Taleisnik. 1993. Changes in water relation parameters under osmotic and salt tolerance in maize and sorghum. Physiology Plantarum, 89(2): 381-387.

12. Farshadfar, A., M.R. Zamani, M. Motalabi and A. Emam jome. 2001. Selected for drought tolerance in chickpea lines. Agricultural Sciences, 32(1): 65-77.

13. Fernandez, G.C.J. 1993. Effective selection criteria for assessing plant stress tolerance. PP: 257-270. In: C. G.KUO (ED), Adaptation of food crops to temperature and water stress, AVRDC, shanhua, Taiwan.

14. Fischer, R.A. and R. Maurer. 1978. Drought tolerance in spring wheat cultivares. I. Grain yield responses. Australian Journal of Agricultural Reaserch, 29: 897- 912.

15. Flowers, T.J., P.F. Troke and A.R. Yeo. 1988. A physiological approach to breeding for resist ance. Inter. Cong. Plant Physiol. New Dehli India. 28: 183-221.

16. Forester, B.P., H. Pakniyat, M. Macaulay, W. Matheson, M.S. Phillips, W.T.B. Thomas and W. Powell. 1994. Variation in the leaf sodium content of the Hordeum vulgare (barley) cultivar Maythorpe and its derived mutant cv. Golden Promis. Heredity, 73: 249-253.

17. Francois, L.E., E.W. Mass, T.J. Donovan and V.L. Youngs. 1986. Effect of salinity on grain yield and quality, vegetative growth and germination of semi-dwarf and durum wheat. Agron. J, 78: 1053-1060.

18. Ghadrian, M., H. Hasanpour, H. Darvishi, H. Mozaffari and T. Rahimi. 2013. Effect of salinity stress on potassium, magnesium, calcium and phosphorus content in mustard herb. National Conference on defective defense in agriculture, Qeshm Island, Iran.

19. Ghannadha, M.R., M. Omidi, R. Abdmishani and K. Poustini. 2005. A study of salt tolerance in genotypes of bread wheat using tissue culture and germination test. Iranian Journal of Agricultural Sciences, 36(1): 75-85.

20. Gholizadeh, A., H. Dehghani, A. Amini and O. Akbarpour. 2018. Investigation of the Genetic Diversity of Iranian Bread Wheat Germplasm for Tolerance to Saline Stress. Journal of Crop Breeding, 10(26):173184.

21. Gorham, J., C. Hardy, R.G. Wyn Jones, L.R. Joppa and C.N. Law. 1987. Chromosomal location of a $\mathrm{K} / \mathrm{Na}$ discriminating character in the D genome of wheat. Theoretical and Applied Genetics, 74: 584-588.

22. Gorji, M. 2008. Effects of the concentration of calcium and potassium in hydroponic nutrient solution on the response of safflower to salinity. M. SC. Thesis, Isfahan university of Technology, Isfahan.

23. Greenway, H. and R. Munns, 1980. Mechanisms of salt tolerance in nonhalophytes. Annual Review of Plant Physiology, 31: 149-190.

24. Hasegawa, P.M., R.A. Bressan and J.K. Zhu. 2000. Plant cellular and molecular responses to high salinity. Annual Review of Plant Physiology, 51: 463-499.

25. Heydarnejad, S. and A. Ranjbar Ferdouei. 2014. Investigating the Effect of Salinity on Some Growth Characteristics and ion accumulation in Seidlitzia rosmarinus L. Desert Ecosystem Engineering Journal, 3(4): $1-10$

26. Hussain, S., R. Munns and A.G. Condon. 2003. Effect of sodium exclusion trait on chlorophyll retention and growth of durum wheat in saline soil. Australian Journal of Agricultural Research, 54(6): 589-597.

27. James, R.A., R.J. Davenport and R. Munns. 2006. Physiological characterization of two genes for Na+ exclosion in durum wheat, Nax1 and Nax2. Pant Physiology, 142: 1537-1547.

28. Lauchli, A. 1984. Salt exclusion: an adaptation of legumes for crops and pastures under saline conditions. In: RC Staples, ed, Salinity Tolerance in Plants: Strategies for Crop Improvement. Wiley, New York, $171-187$ 
29. Mass, E.V. and C.M. Grieve. 1990. Spike and leaf development in salt stressed wheat. Crop Sciences, 30: 1309-1313.

30. Mohammad, M., R. Shibli, M. Ajouni and L. Nimri. 1998. Tomato root and shoot responses to salt stress under different levels of phosphorus nutrition. J. Plant Nutrition. 21: 1667-1680.

31. Molla Heydari Bafghi, R., A. Baghizadeh and G. Mohammadinezhad. 2017. Evaluation of Salinity and Drought Stresses Tolerance in Wheat Genotypes using Tolerance Indices. Journal of Crop Breeding, 9(23): 27-34.

32. Munns, R., G.J. Rebetzke, S. Husain, R.A. James and R.A. Hare. 2003. Genetic control of sodium exclusion in durum wheat. Australian Journal of Agricultural Research, 54: 627-635.

33. Munns, R. and M. Tester. 2008. Mechanismes of salinity tolerance. Annual Review of Plant Biology, 59: 651-681.

34. Netondo, G.W., J.C. Onyango and E. Beck. 2004. Sorghum and salinity: I. Response of growth, Water Relations and Ion Accumulation to NaCl Salinity. Crop Sciences, 44: 797-805.

35. Neumann, P. 1997. Salinity resistance and plant growth revisited. Plant Cell Environment, 20: 11931198 .

36. Pakniyat, H., W.T.B. Thomas, P.D.S. Caligari and B.P. Forester. 1997. Comparision of salt tolerance of GPert and non-GPert barleys. Plant Breeding, 116: 189-191.

37. Pfetffer, C. and H. Bloss. 1988. Growth and nutrition of guayule (Parthenium argentatum) in a saline soil as influenced by vesicular-arbuscular mycorrhiza and phosphorus fertilization. New Phytol, 108(3): 315321.

38. Poustini, K. and M. Beraker. 1991. Interaction of two Triticum vulgar to salinity stress. Journal of Agriculture. SCi. Iran.

39. Poustini, K. and A. Siosemardeh. 2004. Ion distribution in wheat cultivares in responses to salinity stress. Field Crops Research, 85: 125-133.

40. Poustini, K., A. Siosemardeh and M. Ranjbar. 2007. Prolin accumulation as a response to salt stress in 30 wheat (Triticum aestivum L.) cultivares differing in salt tolerance. Genetic Resources and Crop Evolution, 54(5): 925-934.

41. Rashid, A. 1986. Mechanism of salt tolerance in wheat (Triticum aestivum L.) Ph.D. thesis, Department of soil science, University of Agriculture, Faisalabad, Pakistan.

42. Rawson, H.M., R.A. Richards and R. Munns. 1988. An examination of selection criteria for salt tolerance in wheat, barley and triticale. Australian Journal of Agricultural Research, 39(5): 759-772.

43. Renault, S., C. Croser, J.A. Franklin and J.J. Zwiaazek. 2001. Effect of $\mathrm{NaCl}$ and $\mathrm{Na} 2 \mathrm{So} 4$ on red-osier dogwood (Cornus stolonifera Michx). Plant and Soil, 233(2): 261-268.

44. Rosielle, A.A. and J. Hamblin. 1981. Theoretical aspects of selection for yield in stress and non-stress environments. Crop Sciences, 21: 943-946.

45. Sadat Noori, S.A., A. Roustaei and B. Foghi. 2006. Variability of salt tolerance for eleven traits bread wheat in different saline conditions. Agron. J, 5(1): 131-136.

46. Sagi, M., N.A. Savida, N.P. Lvov and S.H. Lips. 1997. Nitrate reductase and molybdenum cofactor in annual ryegrass as affected by salinity and nitrogen source. Physiologia Plantarum, 99(4): 546-553.

47. Schachtman, D.P., R. Munns and M.I. Whitecross. 1991. Variation in sodium exclusion and salt tolerance in Triticum tauschii. Crop Sciences, 31(4): 992-997.

48. Schachtman, D.P. and R. Munns. 1992. Sodium accumulation in leaves of Triticum species that differ in salt tolerance. Australian Journal of Plant Physiology, 19(3): 331-340.

49. Wei, W., P.E. Bilsborrow, P. Hooley, D. Fincham, A.E. Lombi and B.P. Forester. 2003. Salinity induced differences in growth, ion distribution and partitioning in barley between the cultivar Maythorpe and its derived mutant Golden Promise. Plant and Soil, 250(2): 183-191.

50. Weimberg, R.H., H.R. Lerner and A. Poljakoff-Mayber. 1984. Changes in growth and water soluble solute concentration in Sorghum bicolar with sodium and potassium salts. Physiologyia Plantarum, 62(3): $472-480$.

51. Wolf, O., R. Munns, M.L. Tonnet and W.D. Jeschke. 1991. The role of the stem in the partitioning of $\mathrm{Na}+$ and $\mathrm{K}+$ in salt treated barley. Journal of Experimental Botany, 42(6): 697-704.

52. Yeo, A.R. and T.J. Flowers. 1984. Mechanism of salinity resistance in rice and their role as physiological criteria plant breeding. pp: 151-170. In: salinity tolerance in Plants John. Willy. Pub. New York.

53. Yeo, A.R. and T.J. Flowers. 1986. Salinity resistance in rice (Oryza sativa L.) and a pyramiding approach to breeding varieties for saline Soils. Australian Journal of Plant Physiology, 13(1): 161-173.

54. Zhu, J.K. 2001.Plant salt tolerance. Trends Plant Sciences, 6(2): 66-71.

55. Zhu, J.K. 2002. Salt and drought stress signal transducation in plants. Annual Review of Plant Biology, 53: $247-273$. 


\title{
Evaluation of Seed Yield and Accumulation Status of Sodium, Potassium and Magnesium Ions in Different Tissues of Sensitive and Tolerant Wheat (Triticum aestivum L.) Varieties
}

\author{
Amin Baghizadeh', Mahdieh Aram Kasmaie ${ }^{2}$, Ghasem Mohammadi-nejad ${ }^{3}$ and
} Babak Nakhoda ${ }^{4}$

\author{
1- Associate Professor, Department of Biotechnology, Institute of Science and High Technology and Environmental \\ Sciences, Graduate University of Advanced Technology, Kerman, Iran, \\ (Corresponding author: amin_4156@yahoo.com) \\ 2- Graduate M.Sc. Student, Department of Biotechnology, Graduate University of Advanced Technology, Kerman, \\ Iran \\ 3- Associate Professor, Department of Agronomy and Plant Breeding, College of Agriculture, Shahid Bahonar \\ University of Kerman \\ 4- Department of Molecular Physiology, Agricultural Biotechnology Research Institute of Iran, Agricultural \\ Research, Education, and Extension Organization, Karaj, Iran. \\ Received: Jun 28, 2018 \\ Accepted: January 5, 2019
}

\begin{abstract}
Study of biochemical reactions of wheat cultivars to salinity stress can lead to identification of effective mechanisms for salinity tolerance. To determine the ion distribution pattern in wheat, and the effects of salinity stress and ion distribution on grain yield, this study was carried out. This research was conducted in a factorial experiment with two levels of salinity and six tolerant and sensitive wheat cultivars in two replications at research field of Shahid Bahonar University of Kerman on growing season of 2011-2012. The concentration of sodium $\left(\mathrm{Na}^{+}\right)$, potassium $\left(\mathrm{K}^{+}\right)$, magnesium $\left(\mathrm{Mg}^{2}\right)$ ions and $\mathrm{Na}^{+} / \mathrm{K}^{+}$ratio in different tissues of the plant, including flag leaf, spike and root, and grain yield of each plant, were measured. The results of analysis of variance showed that the interaction effects of salinity on the cultivars were significant in most of the traits. The concentration of sodium ions from the roots to the shoots decreased significantly. With increasing salinity, $\mathrm{K}+$ concentration in root of all cultivars decreased significantly. Magnesium concentrations in leaf, spike and root decreased in salinity treatment compared to normal treatment. There was a significant difference between the cultivars in terms of $\mathrm{Na}^{+} / \mathrm{K}^{+}$ratio in all tissues and Moghan 3 cultivar with the highest ratio in spike and root and Arta cultivar with the highest amount in flag leaf, were statistically in one group and other cultivars in the other group. According to the results of simple phenotypic correlation analysis, the sodium ion content in leaf, spike and root tissues had the highest and negative correlation with grain yield per plant. Regarding the reduction of wheat grain yield under salt stress conditions, one way to reduce the damage caused by salinity stress is through ion distribution regulation which can be done in different ways, including selection of tolerant and resistant cultivars against ionic changes.
\end{abstract}

Keywords: Salt stress, Ionic concentration, Grain yield 